www.nature.com/leu

\title{
EDITORIAL
}

\section{NOTCH mutations as prognostic markers in T-ALL}

Leukemia (2010) 24, 2003-2004; doi:10.1038/leu.2010.237

Risk stratification plays a fundamental role in the treatment of acute lymphoblastic leukemia (ALL). Thus, patients harboring clinical features, immunophenotypic markers, cytogenetic or molecular abnormalities that are associated with increased risk of relapse are treated with intensified chemotherapy protocols, while patients classified as low risk are spared from the additional toxicity associated with these treatments.

This strategy has been overly successful in B-precursor ALL, leading to substantial improvements in outcome for specific subsets of high-risk patients. Moreover, in the case of Philadelphia chromosome-positive ALL, combination therapies including BCR-ABL1 tyrosine kinase inhibitors provide a molecularly tailored treatment for this subset of high-risk patients. ${ }^{1}$ For T-ALL in various Berlin-Frankfurt-Munster (BFM)-like treatment protocols, patient stratification has been based primarily on patient response to a glucocorticoid-based prophase therapy as well as minimal residual disease (MRD) analysis.

The recent identification of activating mutations in $\mathrm{NOTCH} 1$ and $F B X W 7$ resulting in constitutively active $\mathrm{NOTCH} 1$ signaling in about $60 \%$ of T-ALL patients opened the question of the prognostic implications of these molecular alterations. ${ }^{2-5}$ Moreover, anti-NOTCH1 therapies hold the promise of providing a molecularly tailored treatment for this disease. ${ }^{2,6}$ Early studies on the prognostic significance of $\mathrm{NOTCH}$ activation in T-ALL showed that NOTCH1 mutations are not associated with poor outcome and suggested that in fact they could be associated with good prognosis. Thus, an original study comprising 157 pediatric T-ALL patients from the pediatric ALL-BFM 2000 study found that NOTCH1 mutations were associated with low levels of MRD and improved relapse-free survival. ${ }^{7}$ However, analysis of a series of 72 pediatric T-ALL patients treated in the Dutch Childhood Oncology Group (DCOG) protocols ALL-7/8 or ALL-9 failed to demonstrate an improved outcome for patients harboring NOTCH1 mutations. ${ }^{8}$ Similarly discrepant results were obtained in adult T-ALL. Thus, analysis of 141 T-ALLs treated in the Lymphoblastic Acute Leukemia in Adults (LALA)-94 $(n=87)$ and the GRAALL-2003 $(n=54)$ trials showed positive prognosis for patients with NOTCH1 and/or FBXW7 mutations. ${ }^{9}$ Yet, these results could not be validated in a series of 88 patients treated in the MRC UKALLXII/ECOGE2993 protocol. $^{10}$ Overall these studies suggested that NOTCH1 mutations could be associated with improved outcome in some series, but that the prognostic impact of NOTCH1 mutations in T-ALL could be influenced by differences in therapy. However, a definitive answer was somehow concealed by methodological differences and the limited sample size of these studies.

Three different studies in this issue of Leukemia revisit the question of the prognosis significance of NOTCH1 and FBXW7 mutations in T-ALL to bring a more conclusive answer to this question. First, a study by Kox and coworkers for an extended series of 301 T-ALL patients treated in the ALL-BFM 2000 study demonstrates that activating mutations in the $\mathrm{NOTCH}$ signaling pathway are associated with improved responses to prophase therapy with glucocorticoids and methotrexate and lower levels of MRD. However, in those patients classified as high risk based on response to prophase therapy and/or MRD, the presence of activating mutations in the NOTCH pathway does not have a positive impact in prognosis.

Next, Clapier et al. report the analysis of 134 patients treated in the EORTC-CLG 58881 and 58951 studies. In this series, NOTCH1 and FBXW7 mutations are associated with improved therapy response and decreased MRD levels. However, this increased response to therapy did not translate to improved outcome. Moreover, in the group of high MRD levels the presence of genetic alterations in the $\mathrm{NOTCH}$ pathway seemed to be associated with worse outcome.

Finally, Zuurbier and coworkers report the study of 72 patient samples from the DCOG ALL7/8 and ALL9 studies and 74 cases treated in the COAL97 clinical trial. The analysis of activated $\mathrm{NOTCH} 1$ protein levels in primary patient samples and microarray expression analysis of NOTCH target genes demonstrate that indeed NOTCH1 and FBXW7 mutations correlate with increased $\mathrm{NOTCH}$ signaling. This study confirms that $\mathrm{NOTCH}$-activating mutations are associated with good prednisone response. However, as in the case of the EORTC-CLG series, this higher sensitivity to chemotherapy did not translate into improved survival.

The association of $\mathrm{NOTCH}$ mutations with increased response to glucocorticoids is indeed a surprising result given that activated NOTCH1 can protect normal thymocytes from glucocorticoidinduced cell death ${ }^{11}$ and that inhibition of $\mathrm{NOTCH}$ signaling with $\gamma$-secretase inhibitors can reverse glucocorticoid resistance in some T-ALL patient samples and cell lines. ${ }^{12,13}$

Overall, these three studies show that $\mathrm{NOTCH}$ activation is associated with improved early therapeutic response. However, this early benefit translates into improved overall survival only in some series, most probably as a result of differences in therapy. In addition, NOTCH1 and FBXW7 mutations fail to predict prognosis among high-risk patients, which is consistent with the presence of NOTCH1 activating mutations in a significant fraction of patients at relapse. As a result, analysis of NOTCH1 and FBXW7 mutations does not seem to improve our current risk-stratification strategies. In this regard, a recent study identifying the absence of biallelic deletion in the TCR $\gamma$ locus as a prognostic marker associated with primary refractory and early-relapse leukemia may provide a better avenue toward developing a molecular risk-stratification approach in T-ALL. ${ }^{14}$

\section{Conflict of interest}

The author declares no conflict of interest.

A Ferrando
${ }^{1,2,3}$
Institute for Cancer Genetics, Columbia University Medical
Center, New York, NY, USA;
${ }^{2}$ Department of Pediatrics, Columbia University Medical
Center, New York, NY, USA and
${ }^{3}$ Department of Pathology, Columbia University Medical
Center, New York, NY,USA
E-mail: af2196@columbia.edu




\section{References}

1 Schultz KR, Bowman WP, Aledo A, Slayton WB, Sather $H$, Devidas $M$ et al. Improved early event-free survival with imatinib in Philadelphia chromosome-positive acute lymphoblastic leukemia: a children's oncology group study. J Clin Oncol 2009; 27: 5175-5181.

2 Weng AP, Ferrando AA, Lee W, Morris IV JP, Silverman LB, Sanchez-Irizarry $\mathrm{C}$ et al. Activating mutations of $\mathrm{NOTCH} 1$ in human T cell acute lymphoblastic leukemia. Science 2004; 306: 269-271.

3 Sulis ML, Williams O, Palomero T, Tosello V, Pallikuppam S, Real PJ et al. NOTCH1 extracellular juxtamembrane expansion mutations in T-ALL. Blood 2008; 112: 733-740.

4 Thompson BJ, Buonamici S, Sulis ML, Palomero T, Vilimas T, Basso G et al. The SCFFBW7 ubiquitin ligase complex as a tumor suppressor in T cell leukemia. J Exp Med 2007; 204: 1825-1835.

5 O'Neil J, Grim J, Strack P, Rao S, Tibbitts D, Winter C et al. FBW7 mutations in leukemic cells mediate $\mathrm{NOTCH}$ pathway activation and resistance to gamma-secretase inhibitors. J Exp Med 2007; 204: 1813-1824.

6 Palomero T, Barnes KC, Real PJ, Bender JL, Sulis ML, Murty VV et al. CUTLL1, a novel human T-cell lymphoma cell line with $t(7 ; 9)$ rearrangement, aberrant NOTCH1 activation and high sensitivity to gamma-secretase inhibitors. Leukemia 2006; 20: 1279-1287.

7 Breit S, Stanulla M, Flohr T, Schrappe M, Ludwig WD, Tolle G et al. Activating $\mathrm{NOTCH} 1$ mutations predict favorable early treatment response and long-term outcome in childhood precursor T-cell lymphoblastic leukemia. Blood 2006; 108: 1151-1157.
8 van Grotel M, Meijerink JP, Beverloo HB, Langerak AW, Buys-Gladdines JG, Schneider $P$ et al. The outcome of molecular-cytogenetic subgroups in pediatric T-cell acute lymphoblastic leukemia: a retrospective study of patients treated according to DCOG or COALL protocols. Haematologica 2006; 91: 1212-1221.

9 Asnafi V, Buzyn A, Le Noir S, Baleydier F, Simon A, Beldjord K et al. NOTCH1/FBXW7 mutation identifies a large subgroup with favorable outcome in adult T-cell acute lymphoblastic leukemia (T-ALL): a Group for Research on Adult Acute Lymphoblastic Leukemia (GRAALL) study. Blood 2009; 113: 3918-3924.

10 Mansour MR, Sulis ML, Duke V, Foroni L, Jenkinson S, Koo K et al. Prognostic implications of $\mathrm{NOTCH} 1$ and $\mathrm{FBXW} 7$ mutations in adults with T-cell acute lymphoblastic leukemia treated on the MRC UKALLXII/ECOG E2993 protocol. J Clin Oncol 2009; 27: 4352-4356.

11 Deftos ML, He YW, Ojala EW, Bevan MJ. Correlating notch signaling with thymocyte maturation. Immunity 1998; 9: 777-786.

12 Real PJ, Tosello V, Palomero T, Castillo M, Hernando E, de Stanchina $\mathrm{E}$ et al. Gamma-secretase inhibitors reverse glucocorticoid resistance in T cell acute lymphoblastic leukemia. Nat Med 2009; 15: 50-58.

13 Real PJ, Ferrando AA. NOTCH inhibition and glucocorticoid therapy in T-cell acute lymphoblastic leukemia. Leukemia 2009; 23: $1374-1377$.

14 Gutierrez A, Dahlberg SE, Neuberg DS, Zhang J, Grebliunaite R, Sanda T et al. Absence of biallelic TCR \{gamma\} deletion predicts early treatment failure in pediatric T-cell acute lymphoblastic leukemia. J Clin Oncol 2010; 28: 3816-3823. 